\title{
Quantum tunneling and stochastic resonance
}

\author{
Milena Grifoni, Ludwig Hartmann, Sabine Berchtold, and Peter Hänggi \\ Institut für Physik, Universität Augsburg, Memminger Straße 6, D-86135 Augsburg, Germany
}

(Received 21 December 1995)

\begin{abstract}
Stochastic resonance (SR) occurs in nonlinear dynamical systems when the response to a weak coherent input is enhanced by the presence of noise. While classical SR presently is an intensely studied phenomenon, the role of quantum fluctuations has only started to be explored. We study SR in the temperature range where quantum tunneling corrections to the classical rate of activation are relevant. For a particle subject to moderateto-large friction, we show that the semiclassical SR can be enhanced considerably by quantum fluctuations, as compared to the predictions of a classical analysis. [S1063-651X(96)10406-2]
\end{abstract}

PACS number(s): 05.40. $+\mathrm{j}, 05.30 .-\mathrm{d}$, 03.65.Sq, 33.80.Be

\section{INTRODUCTION}

Stochastic resonance (SR) is the process whereby noise operates on a bistable system enhancing the response to a weak coherent input. Since its discovery in 1981 [1], this intriguing phenomenon has been the object of many investigations [2,3], stimulated by its experimental demonstration in biological [4] and physical [5] systems. The archetypal model for SR is that of a particle of mass $M$ moving in a double-well potential while coupled to a heat bath at temperature $T$ and subject to a time-dependent periodic force. Classically, the resonance condition is assumed when the thermal hopping frequency is near the frequency of the modulation [6-9]. In the presence of memory effects, the classical escape rate out of a metastable well reads, for moderate-to-strong friction [10],

$$
\Gamma_{\mathrm{cl}}=\frac{\omega_{a}}{2 \pi} \frac{\omega_{R}}{\omega_{b}} \exp \left(\frac{-V_{b}}{k_{B} T}\right)
$$

Here, $\omega_{a}=\left[V^{\prime \prime}\left(q_{a}\right) / M\right]^{1 / 2}$ is the angular frequency of small oscillations about the metastable minimum at $q_{a}$, $\omega_{b}=\left[-V^{\prime \prime}\left(q_{b}\right) / M\right]^{1 / 2}$, and $V_{b}$ are the angular barrier frequency and barrier height located at $q_{b}$ (see also Fig. 1). Finally, the friction-renormalized angular barrier frequency $\omega_{R}$ is defined below in Eq. (1.3). It is readily seen that the classical transmission factor $\omega_{R} / \omega_{b}<1$ determines the difference between the transition-state-theory result and the correct classical rate due to diffusive recrossing of the barrier. Equation (1.1) generalizes the pioneering work by Kramers [11] on the effects of frequency-independent friction on the escape rate [see Eq. (1.4)], to include frequency-dependent damping. The Arrhenius law (1.1) predicts a vanishing rate $\Gamma$ as the temperature $T$ approaches absolute zero. However, quantum mechanics allows for the possibility of crossing the barrier through quantum tunneling, thus leading to a finite (quantum) rate at zero temperature.

Tunneling transitions dominate over thermally activated transitions below a crossover temperature $T_{0}$ (see Fig. 2). A simple criterion for the crossover temperature $T_{0}$ was given by Gol'danskii in 1959 [12]. For a parabolic barrier with barrier frequency $\omega_{b}$, he found $T_{0}=\hbar \omega_{b} / 2 \pi k_{B}$. The Gol'danskii criterion, however, disregards the environmental influence upon the tunneling rate. In order to fully take into account the influence of dissipation and thermal fluctuation on the tunneling rates, a functional integral approach has been employed in [13-15], while an alternative derivation based on the periodic orbit approach is discussed in $[16,17]$. It is found that

$$
T_{0}=\hbar \omega_{R} / 2 \pi k_{B}
$$

where $\omega_{R}$ is a dissipation-renormalized frequency, which is given by the largest positive solution of the equation

$$
\omega_{R}^{2}+\omega_{R} \hat{\gamma}\left(\omega_{R}\right)=\omega_{b}^{2},
$$

with $\hat{\gamma}(\omega)$ being the Laplace transform of the friction kernel $\gamma(t)$ appearing in the classical equation of motion [see below Eq. (2.4)]. This relation holds independent of the detailed shape of the potential provided that it is parabolic in the vicinity of the barrier top. In the case of frequencyindependent damping, i.e., $\hat{\gamma}(\omega)=\gamma$, one has

$$
\omega_{R}=\left(\omega_{b}^{2}+\gamma^{2} / 4\right)^{1 / 2}-\gamma / 2 .
$$

It should be noted that the crossover temperature may be quite large and can reach for some physical and chemical systems values larger than $100 \mathrm{~K}[17,18]$. On the other hand,

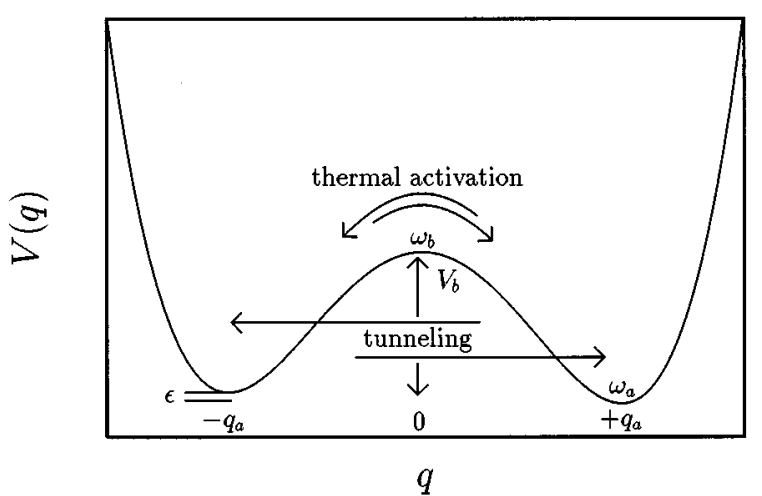

FIG. 1. Thermal and quantum fluctuations influence the escape rates out of the metastable wells of an asymmetric bistable potential. 


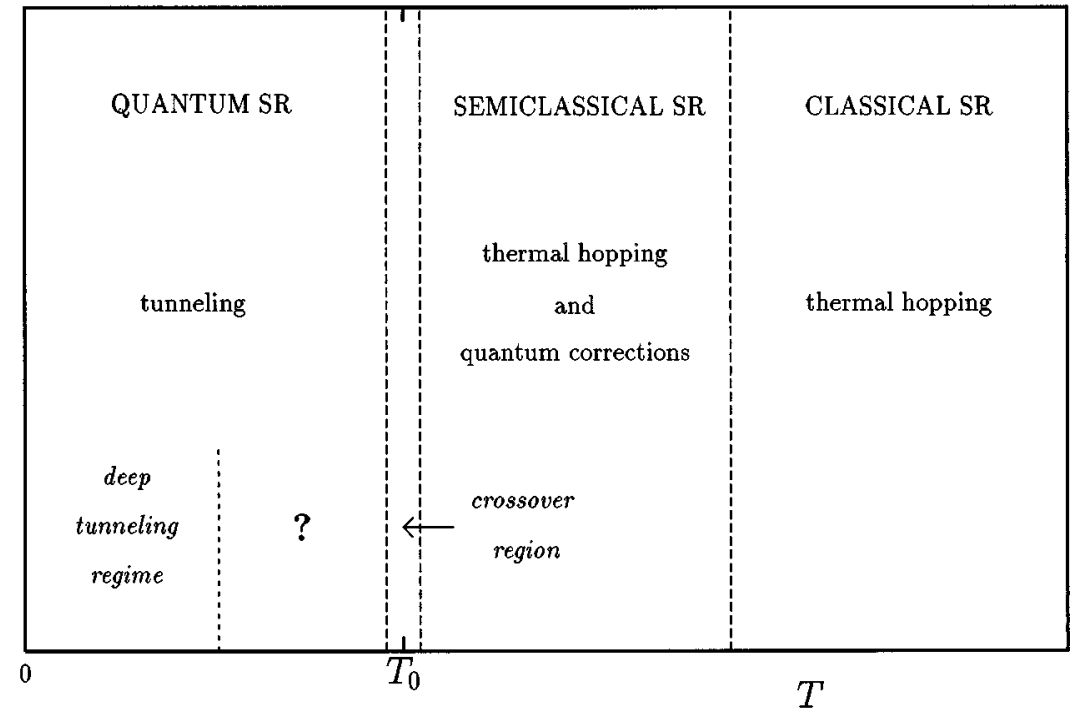

FIG. 2. Dominant escape mechanism out of a metastable potential, and corresponding regimes for SR, depicted as a function of temperature. $T_{0}$ denotes the crossover temperature below which quantum tunneling dominates over thermally activated hopping events. Because $T_{0}$ is a function of the dissipation mechanism, the relative size of the corresponding regions varies with the dissipation strength. In the region marked by a question mark, quantum SR has, up to now, not been investigated theoretically. in Josephson systems where both classical SR [19] and quantum corrections $[17,20,21]$ have been observed, it can be in the $\mathrm{mK}$ region.

The role of quantum fluctuations on SR has only started to be explored. As a matter of fact, the quantum tunneling mechanism for the escape rate, and hence for SR itself, is strongly dependent on temperature (see Fig. 2). Prior studies $[22,23]$ on the effects of quantum noise on SR addressed the regime of very low temperatures $T \ll T_{0}$, where thermal hopping events can be neglected.

The focus of this work is on the effect of quantum fluctuations in the opposite regime $T>T_{0}$, where quantum tunneling is not the dominant escape mechanism, but leads to significant quantum corrections of the classical rate of activation (temperature regime of semiclassical SR, as denoted in Fig. 2). Because $T_{0}$ is a function of the dissipation mechanism, the relative size of the corresponding regions varies with the dissipation strength. In particular, the semiclassical region may extend far above $T_{0}$ (cf. Figs. 3-6). In the present work, we leave out only the very narrow temperature region around $T_{0}$, determined by the condition
$\left|T / T_{0}-1\right| \leqslant\left(\hbar \omega_{R} / V_{b}\right)^{1 / 2}$ (and denoted "crossover region", in Fig. 2), where the evaluation of the escape rates requires going beyond the semiclassical treatment discussed in Sec. III.

In the investigated temperature regime $T>T_{0}$, the presence of the additional quantum "channel" for barrier crossing results in a quantum correction factor that merely multiplies the classical rate of activation [see Eq. (3.1) below]. We find then that (for moderate-to-strong damping) the semiclassical SR can become enhanced up to two orders of magnitude, as compared to the predictions for SR based on a pure classical SR analysis.

\section{THE BISTABLE MODEL FOR SR}

To investigate semiclassical SR, we consider a particle of mass $M$ moving in an asymmetric bistable potential $V(q)$ (see Fig. 1), while coupled to a heat bath and subject to a time-dependent periodic force $f(t)=-A \cos \Omega t$. The poten-

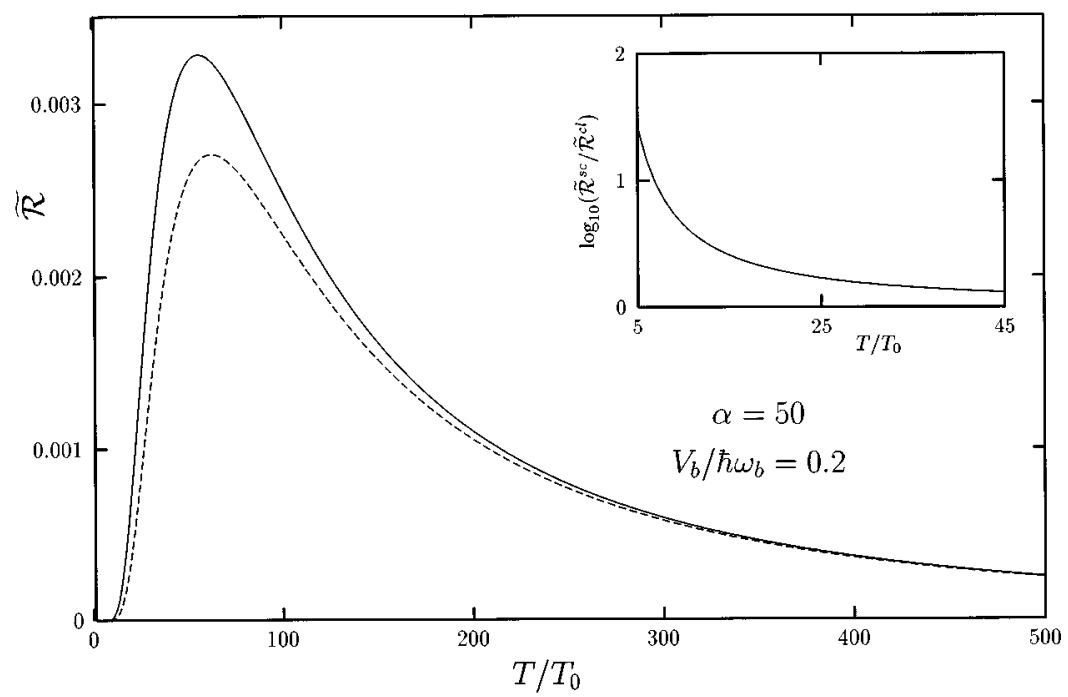

FIG. 3. Amplification vs temperature of the semiclassical scaled signal-to-noise-ratio $\widetilde{\mathcal{R}^{s c}}$ as influenced by quantum fluctuations (solid line). For comparison, the classical signal-to-noiseratio is also depicted (dashed line). The inset shows that the enhancement of the semiclassical $\widetilde{\mathcal{R}^{s c}}$, as compared to the classical one $\widetilde{\mathcal{R}^{c l}}$, can reach two orders of magnitude. 


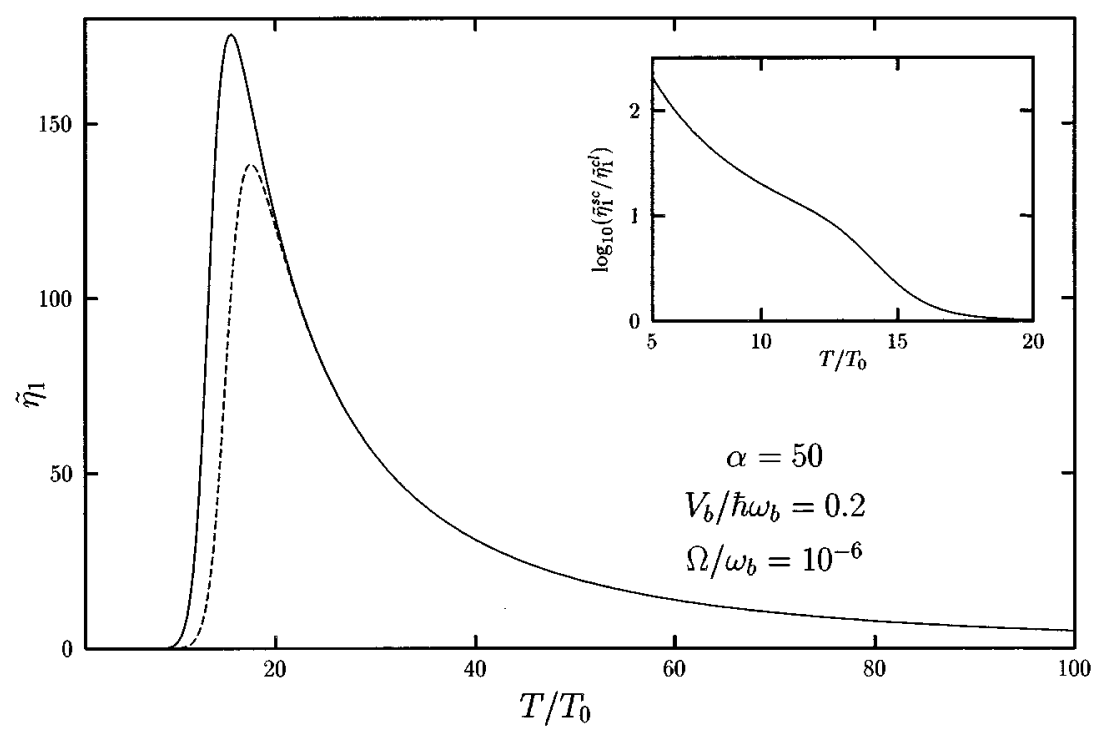

FIG. 4. Amplification vs temperature of the semiclassical scaled fundamental amplitude $\widetilde{\eta}_{1}^{s c}$ which accounts for quantum tunneling fluctuations (solid line). For comparison, the classical power amplitude $\widetilde{\eta}_{1}^{c l}$ is also drawn (dashed line). The inset shows that quantum tunneling can enhance the semiclassical $\widetilde{\eta}_{1}^{s c}$, with respect to the classical one $\tilde{\eta}_{1}^{c l}$, up to two orders of magnitude.

tial is characterized by an asymmetry energy $\epsilon \geqslant 0$ and becomes symmetric $V(q)=V(-q)$ when $\epsilon=0$. The two metastable minima are located at $\pm q_{a}$ and the maximum is at $q_{b}=0$. Due to the asymmetry of the potential, the barrier height to be surmounted by a classical particle located at $\pm q_{a}$ is $E_{ \pm}=V_{b} \mp \epsilon / 2$, depending on whether the particle is in the left or right well, respectively. Finally, the concept of metastability makes sense only when the barrier is large enough so that the forward $\Gamma_{+}$and backward $\Gamma_{-}$escape rates out of the metastable states are very small compared with all the other characteristic rate scales of the system dynamics. In particular, because the angular frequency $\omega_{a}$ describes the time scale for decay within a metastable well, the activation energies $E_{+}$are to be large enough compared to the thermal energy $k_{B} T$ to ensure that the condition $\omega_{a} \gg \Gamma_{ \pm}$is fulfilled [17].

The heat bath is assumed to be representable as a set of harmonic oscillators interacting bilinearly with the particle $[24,25]$, so that the Hamiltonian takes the form

$$
\begin{aligned}
H= & \frac{p^{2}}{2 M}+V(q)+\sum_{i=1}^{N}\left[\frac{p_{i}^{2}}{2 m_{i}}+\frac{m_{i} \omega_{i}^{2}}{2}\left(x_{i}-\frac{c_{i}}{m_{i} \omega_{i}^{2}} q\right)^{2}\right] \\
& -q f(t),
\end{aligned}
$$

where the quantum thermal noise $\xi(t)$ is fully characterized by a zero average $\langle\xi(t)\rangle_{0}=0$ and by the correlation function

$$
\langle\xi(t) \xi(0)\rangle_{0}=\frac{\hbar M}{\pi} \int_{0}^{\infty} d \omega \omega \widetilde{\gamma}(\omega) \frac{\cosh [\omega(\hbar \beta / 2-i t)]}{\sinh (\omega \hbar \beta / 2)},
$$

with $\beta=1 / k_{B} T$. Here \langle\rangle$_{0}$ denotes the statistical average over the bath degrees of freedom with all the coupling constants $c_{i}$ set to zero and in the absence of the external force $f(t)$. Finally, the friction coefficient $\widetilde{\gamma}(\omega)=\int_{0}^{\infty} d t \cos (\omega t) \gamma(t)$ appearing in Eq. (2.2) is the real part of the Fourier transform of the time-dependent memory friction

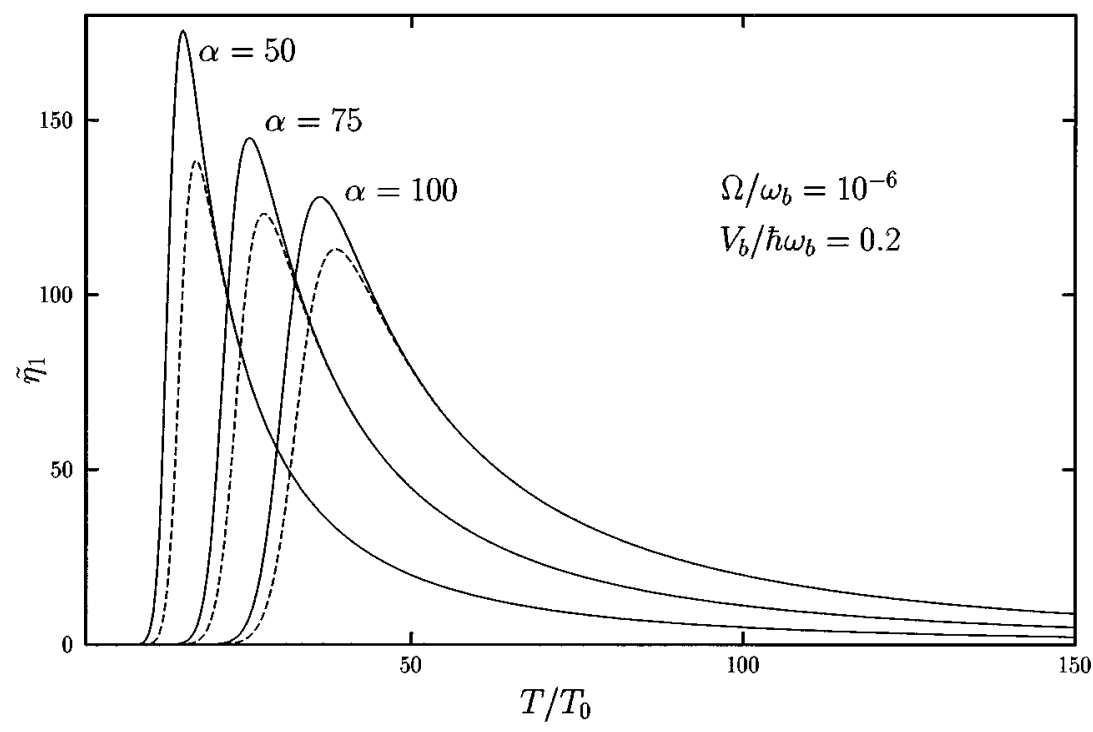

FIG. 5. Amplification vs temperature of the semiclassical power amplitude $\widetilde{\eta}_{1}^{s c}$ for different coupling strengths $\alpha=\gamma / 2 \omega_{b}$ (solid lines). For strong damping, the effects of quantum fluctuations extend well above the crossover temperature $T_{0}$. For comparison, the classical power amplitudes are also drawn (dashed lines). 


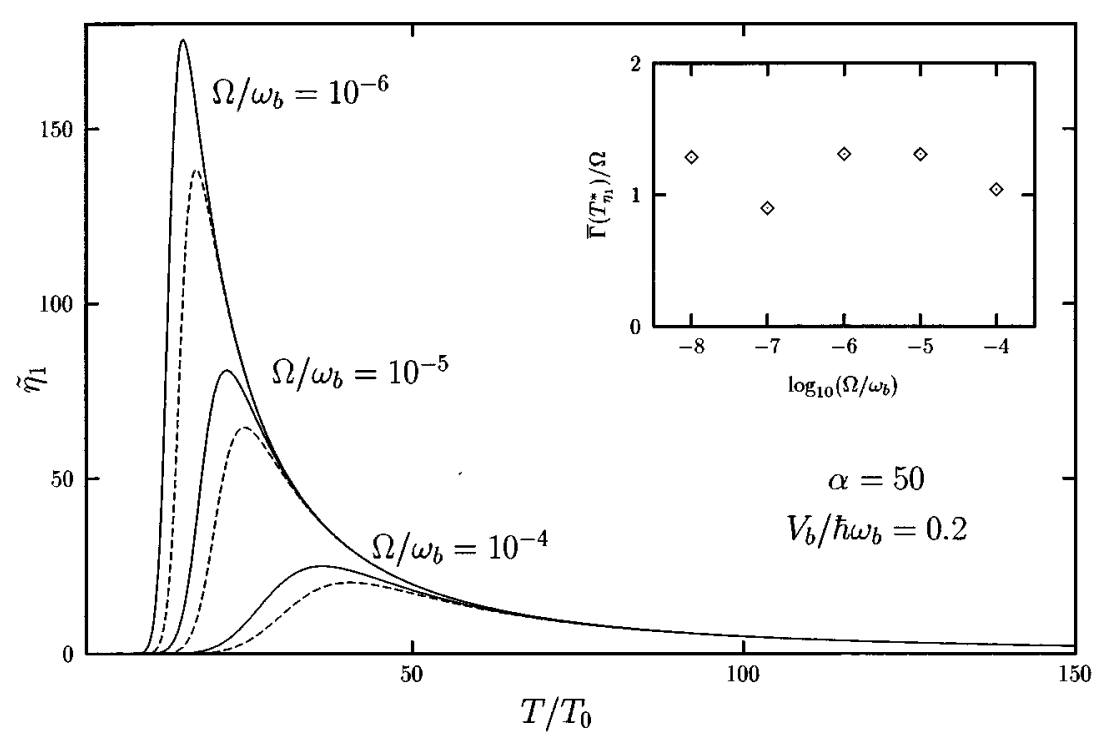

FIG. 6. Amplification vs temperature of the semiclassical amplitude $\widetilde{\eta}_{1}^{s c}$ for different driving frequencies $\Omega$ (solid lines). For comparison, the classical power amplitudes are also plotted (dashed lines). The inset shows that the temperature maximum $T_{\eta_{1}}^{*}(\Omega)$ of $\widetilde{\eta}_{1}$ is approximately determined by the condition $\bar{\Gamma}\left(T_{\eta_{1}}^{*}\right) \approx \Omega$, over a range of four orders of magnitude spanned by $\Omega$.

$$
\gamma(t)=\frac{1}{M} \sum_{i} \frac{c_{i}^{2}}{m_{i} \omega_{i}^{2}} \cos \left(\omega_{i} t\right)
$$

The density and coupling constants of the environment are chosen in such a way that the particle obeys the classical, generalized Langevin equation of motion with memory friction [26],

$$
M \ddot{q}+\frac{\partial V}{\partial q}+M \int_{-\infty}^{t} d s \gamma(t-s) \dot{q}(s)=\xi_{c}(t)+f(t),
$$

where the frequency-dependent damping coefficient $\widetilde{\gamma}(\omega)$ and the force-force correlation function (2.2) satisfy in the classical limit $\hbar \beta \omega \rightarrow 0$ the Green-Kubo formula

$$
\widetilde{\gamma}(\omega)=\frac{1}{M k_{B} T} \int_{0}^{\infty} d t e^{i \omega t}\left\langle\xi_{c}(t) \xi_{c}(0)\right\rangle_{0},
$$

and $\xi_{c}(t)$ denotes the thermal classical noise. Hence, the thermal noise is characterized by the temperature $T$ of the thermal bath and by the coupling $\gamma$ of the bistable system to the environment. It should be noted that, while in the classical regime the Arrhenius factors for the escape rates $\Gamma_{ \pm}$are independent of damping, and only the attempt frequencies are modified [cf. Eq. (1.1)], in the quantum regime the prefactor of the rate and the exponent as well crucially depend on the strength $\widetilde{\gamma}$ of the dissipative mechanism. Finally, the expectation value with respect to the full Hamiltonian (2.1) of the particle's position

$$
P(t)=\langle q(t)\rangle
$$

is considered to be the output of the system when the external time-dependent periodic force $f(t)=A \cos \Omega t$ modulating the particle's position is applied. In particular, the averaged power spectrum $\bar{S}(\omega)$

$$
\bar{S}(\omega)=\int_{-\infty}^{+\infty} d \tau e^{i \omega \tau} \bar{C}(\tau)=S_{N}(\omega)+S^{(\text {as })}(\omega),
$$

defined as the Fourier transform of the averaged correlation function $\bar{C}(\tau)$,

$$
\bar{C}(\tau)=\frac{\Omega}{2 \pi} \int_{0}^{2 \pi / \Omega} d t \frac{1}{2}\langle q(t+\tau) q(t)+q(t) q(t+\tau)\rangle,
$$

is the quantity of interest to investigate SR [27]. For a timeperiodic perturbation, the power spectrum results in the sum of two contributions, where $S_{N}$ represents, in the absence of a signal, the broadband "noise" background, possessing a Lorentzian hump at $\omega=0$. We shall denote this contribution by $S_{N}^{(0)}$. In the presence of the signal, $S_{N}$ is obtained as a product of the Lorentzian hump with a correction factor (of order unity for weak signals) describing the influence of the signal $[6,8]$. The "asymptotic', contribution $S^{(\text {as })}(\omega)$ is given by the sum of $\delta$ spikes at integer multiples $\omega=n \Omega$ of the signal frequency, reflecting the fact that, for times $t$ large compared to the time scale of the transient dynamics, the motion acquires the periodicity of the external perturbation. To be definite, $P(t)$ and $\bar{C}(\tau)$ reach the asymptotic values $[6,23]$

$\lim _{t \rightarrow \infty} P(t)=P^{(\text {as })}(t)=\sum_{m=-\infty}^{\infty} P_{m}(\Omega, A) e^{-i m \Omega t}$,
$\lim _{\tau \rightarrow \infty} \bar{C}(\tau)=C^{(\text {as })}(\tau)=\sum_{m=-\infty}^{\infty}\left|P_{m}(\Omega, A)\right|^{2} e^{-i m \Omega \tau}$.

Thus, the amplitudes $\left|P_{m}\right|$ of the harmonics of $P(t)$ determine the weights of the $\delta$ spikes of the averaged spectral power density in the asymptotic state $S^{(\text {as) }}(\omega)$ via the relation

$$
S^{(\mathrm{as})}(\omega)=2 \pi \sum_{m=-\infty}^{\infty}\left|P_{m}(\Omega, A)\right|^{2} \delta(\omega-m \Omega) .
$$

The two quantities that have been examined in the literature to investigate SR are the power amplitude $\eta_{1}$ in the first 
frequency component of $S^{(\text {as) }}(\omega)[3,6]$ and the ratio $\mathcal{R}$ of $\eta_{1}$ to the power spectrum $S_{N}^{(0)}(\omega)$ of $q(t)$ in the absence of signal evaluated at the external frequency, the so called signal-to-noise ratio (SNR) $[2,3,8]$, i.e.,

$$
\begin{gathered}
\eta_{1}(\Omega, A):=4 \pi\left|P_{1}(\Omega, A)\right|^{2}, \\
\mathcal{R}:=4 \pi\left|P_{1}(\Omega, A)\right|^{2} / S_{N}^{(0)}(\Omega) .
\end{gathered}
$$

By definition, $\eta_{1}$ has the dimension of a length squared, while $\mathcal{R}$ has the dimension of a frequency. Thus, to investigate the interplay between noise and the coherent driving input giving rise to the phenomenon of stochastic resonance, we shall consider the two dimensionless quantities, the scaled power amplification $\widetilde{\eta}_{1}$, and the scaled signal-tonoise ratio $\widetilde{\mathcal{R}}$. They read

$$
\widetilde{\eta}_{1}(\Omega, A)=\frac{\eta_{1}(\Omega, A)}{\left(A q_{a}^{2} / V_{b}\right)^{2}}, \quad \widetilde{\mathcal{R}}=\frac{\left(\mathcal{R} / \omega_{b}\right)}{\left(A q_{a} / V_{b}\right)^{2}} .
$$

Which one of the two quantities is the most appropriate to investigate SR depends on experimental realization $[2,3,19]$.

\section{A. Linear-response theory for SR}

Because the main theme of SR is the stochastic enhancement of the response to a weak coherent input, we shall develop in this section a theory for SR based on Kubo's linear-response theory and on fluctuation-dissipation theorem (FDT) [28]. The results for the spectral amplification $\eta_{1}$ and for the SNR are expressed in such a way as to be independent of the precise dynamics of the system (being dominated by quantum tunneling transitions or/and by thermally activated hopping events), assuming that the resulting escape rates at thermal equilibrium (i.e., for the undriven dissipative bistable system) are known. This will enable us to compare the predictions for SR above the crossover temperature $T_{0}$ which would be obtained using a "classical approximation" for the rates [cf. Eq. (1.1)], with those obtained using decay rates corrected for quantum tunneling as in Eq. (3.1) (see below).

In the linear-response approximation, only the harmonics $0, \pm 1$ of $P^{(\text {as) }}(t)$ in Eq. (2.9) are different from zero, $P_{0}$ being just the thermal equilibrium value $P_{\text {eq }}$ in the absence of driving, and $P_{ \pm 1}=(A / 2) \widetilde{\chi}( \pm \Omega)$ being related by $\mathrm{Ku}-$ bo's formula to the linear susceptibility $\widetilde{\chi}(\Omega)$,

$$
\widetilde{\chi}(\Omega)=\frac{1}{i \hbar} \int_{-\infty}^{+\infty} d \tau e^{i \Omega \tau} \theta(\tau)\langle[q(\tau), q(0)]\rangle_{\beta},
$$

where \langle\rangle$_{\beta}$ indicates the evaluation of correlation functions in thermal equilibrium, that is, in the absence of driving. Further, $i\langle[q(\tau), q(0)]\rangle_{\beta} / \hbar$ becomes in the classical case the correlation function $-\beta\langle q(0) \dot{q}(\tau)\rangle_{\beta}$. Finally, because the linear susceptibility is related to the power spectrum in thermal equilibrium by the fluctuation-dissipation theorem [28], we end up with

$$
\eta_{1}(\Omega, A)=\pi A^{2}|\widetilde{\chi}(\Omega)|^{2},
$$

$$
\mathcal{R}=\pi A^{2} \frac{1}{\hbar \operatorname{coth}(\beta \hbar \Omega / 2)} \frac{|\widetilde{\chi}(\Omega)|^{2}}{\operatorname{Im} \widetilde{\chi}(\Omega)} .
$$

Thus, for weak external signals, computation of the power amplitude $\eta_{1}$ or of the signal-to-noise ratio $\mathcal{R}$ reduces to the evaluation of correlation functions in thermal equilibrium. It should be noted that the above relationships are valid independently whether the relaxation occurs via quantum or via classical decay.

In order to evaluate the linear susceptibility, a knowledge of the equlibrium dynamics is required. In doing so, we shall derive our results within a two-state description of the system dynamics, introducing the probabilities $n_{\mathrm{L}, \mathrm{R}}$ for the system to be in the left $\left(n_{\mathrm{L}}\right)$ or right $\left(n_{\mathrm{R}}\right)$ well of the bistable potential. For a continuous system, $n_{\mathrm{L}, \mathrm{R}}$ are defined in terms of the probability density $p(q, t)$ for the particle's position as

$$
n_{\mathrm{L}}(t)=1-n_{\mathrm{R}}(t)=\int_{-\infty}^{q_{b}} d q p(q, t) .
$$

One then finds that the average value in Eq. (2.6) is simply $P(t)=q_{a}\left[n_{\mathrm{R}}(t)-n_{\mathrm{L}}(t)\right]$ and obeys, for a classical or a semiclassical dynamics, the Markovian rate equation

$$
\dot{P}(t)=-\bar{\Gamma}\left[P(t)-P_{\mathrm{eq}}\right],
$$

with $\bar{\Gamma}=\Gamma_{+}+\Gamma_{-}$being the sum of the forward and backwards rates $\Gamma_{+}$and $\Gamma_{-}$, respectively, and $P_{\mathrm{eq}}=\left(\Gamma_{+}-\Gamma_{-}\right) / \bar{\Gamma}$. Information about the detailed form of the potential is still contained in the averaged rate $\bar{\Gamma}$. In the deep quantum regime $T \ll T_{0}$ (cf. Fig. 2) the same set of equations holds whenever incoherent tunneling dominates the dynamics, as it always holds true for strong enough damping or sufficiently high temperatures $[29,30]$. Correspondingly, in the limit $\hbar \beta \Omega / 2 \ll 1$, the (classical or quantum) linear susceptibility $\widetilde{\chi}(\Omega)$ exhibits a quasielastic Lorentzian peak of amplitude $b(T)=4\left(q_{a}^{2} / k_{B} T\right) \Gamma_{+} \Gamma_{-} / \bar{\Gamma}^{2}$ and width $\bar{\Gamma}$. It reads

$$
\widetilde{\chi}(\Omega)=b(T) \frac{1}{1-i \Omega \bar{\Gamma}^{-1}}+O(\hbar \beta \Omega)^{2} .
$$

Finally, whenever the backward and forward rates are related by the detailed balance condition $\Gamma_{-}=\Gamma_{+} \exp \left(-\epsilon / k_{B} T\right)$, we obtain for the scaled power amplitude $\widetilde{\eta}_{1}$ the result

$$
\widetilde{\eta}_{1}(\Omega)=\pi\left(\frac{V_{b}}{k_{B} T}\right)^{2} \frac{1}{\cosh ^{4}\left(\epsilon / 2 k_{B} T\right)} \frac{\bar{\Gamma}^{2}}{\Omega^{2}+\bar{\Gamma}^{2}} .
$$

Likewise, consistent with condition $\hbar \beta \Omega / 2 \ll 1$, the cotangent hyperbolicus in Eq. (2.16) can be approximated as the inverse of its argument, and the scaled signal-to-noise-ratio $\widetilde{\mathcal{R}}$ becomes effectively independent of the external frequency $\Omega$. One finds

$$
\widetilde{\mathcal{R}}=\frac{\pi}{2}\left(\frac{V_{b}}{k_{B} T}\right)^{2} \frac{\bar{\Gamma} / \omega_{b}}{\cosh ^{2}\left(\epsilon / 2 k_{B} T\right)} .
$$

Several features of the results in Eqs. (2.20), (2.21) are worth commenting on. 


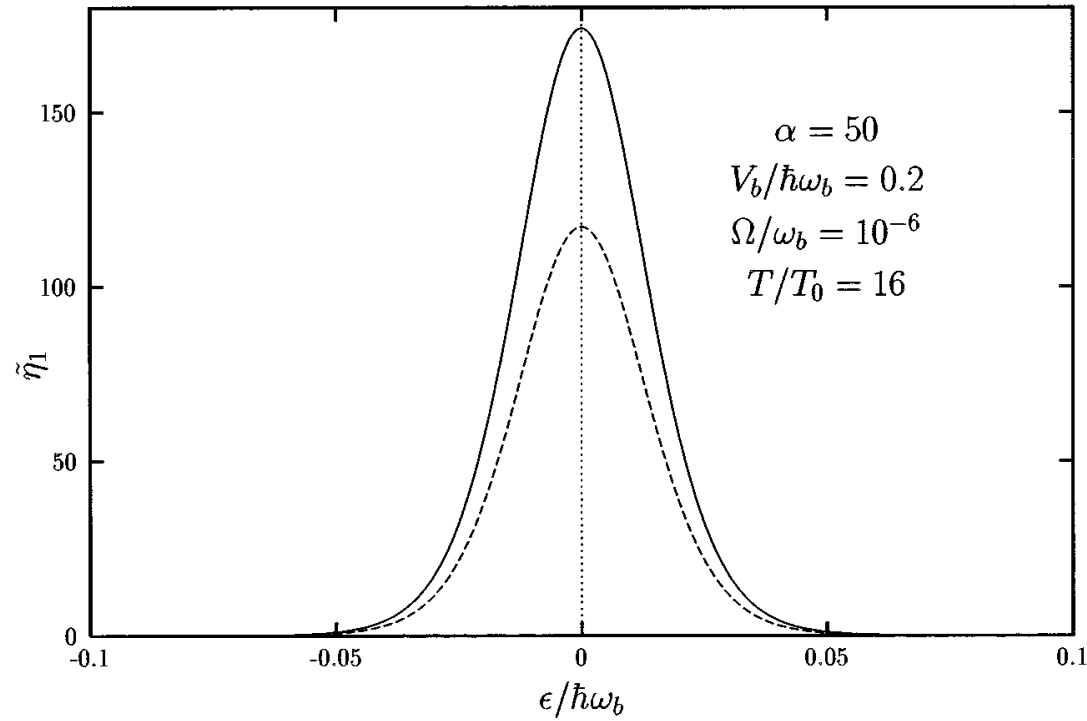

FIG. 7. Semiclassical amplitude $\tilde{\eta}_{1}^{s c}$ vs the asymmetry of the potential (solid line) compared to the classical power amplitude (dashed line). As in the classical case, $\widetilde{\eta}_{1}^{s c}$ is maximal for a symmetric bistable potential $(\epsilon=0)$. (i) Within a two-state description of the incoherent (undriven) dynamics, the linear-response theory developed in this section effectively reduces the study of SR to the computation of the transitions rates $\Gamma_{+}$or $\Gamma_{-}$in thermal equilibrium.

(ii) By construction, a linear-response approximation holds independent of whether the coherent applied signal $A \cos (\Omega t)$ involves adiabatic or nonadiabatic frequencies. Hence, Eqs. (2.15) and (2.16) hold for any driving frequency $\Omega$. On the other hand, while the expression (2.19) for the linear susceptibility $\widetilde{\chi}(\Omega)$ becomes exact in the classical limit, the condition $\hbar \beta \Omega / 2 \ll 1$ requires some care in the semiclassical and deep quantum regimes and may lead to restrictions on the values of the applied driving frequency $\Omega$, as discussed in the next section. Whenever the condition $\hbar \beta \Omega / 2 \ll 1$ is not fullfilled, the linear susceptibility $\widetilde{\chi}(\Omega)$ (and hence $\widetilde{\eta}_{1}$ and $\widetilde{\mathcal{R}}$ ) exhibits a more complicated dependence on the frequency $\Omega$, as determined by the fluctuationdissipation theorem [28] and by the Kramers-Kronig relationships between its real and imaginary parts [see Refs. $[22,23]$ for a discussion of SR in the deep quantum regime].

(iii) Because Eqs. (2.20), (2.21) hold independent of whether the escape mechanism is classical or quantum, some general features of SR can be discussed. For the case of weak external signals considered in Eqs. (2.20) and (2.21), both the scaled amplitude $\widetilde{\eta}_{1}$ and the scaled signal-to-noise ratio $\widetilde{\mathcal{R}}$ are independent of the external strength $A$, but only $\widetilde{\eta}_{1}$ is still a function of the external frequency $\Omega$. Hence, the position $T_{\mathcal{R}}^{*}$ of the temperature maximum of the scaled SNR effectively depends only on intrinsic parameters of the bistable system, such as the barrier height $V_{b}$, the asymmetry $\epsilon$, the frequencies $\omega_{b}$ and $\omega_{a}$, and the friction coefficient. By contrast, the temperature maximum $T_{\eta_{1}}^{*}$ of the scaled power amplitude $\widetilde{\eta}_{1}$ is roughly determined by the condition $\bar{\Gamma}\left(T_{\eta_{1}}^{*}\right) \approx \Omega$ (cf. inset of Fig. 6). This implies that SR for $\tilde{\eta}_{1}$ can be externally controlled by varying the applied driving frequency $\Omega$.

(iv) On the same basis as (iii), the generality of Eqs. $(2.20,2.21)$ implies that the differences between classical, semiclassical or quantum SR (cf. Fig. 2) are determined solely by the explicit temperature dependence of the escape rates $\Gamma_{ \pm}$. In particular, the classical [cf. Eq. (1.1)] and the semiclassical [cf. Eqs. (3.1), (3.2), (3.3)] transition rates decay exponentially as the temperature decreases. This, together with the (classical and semiclassical) condition $V_{b} \gg k_{B} T$ necessary for a separation of time scales, implies that the SR maxima are determined by the competition between this exponential decay and the algebraic divergence $\left(k_{B} T\right)^{-2}$ in $\widetilde{\eta}_{1}$ or in $\widetilde{\mathcal{R}}$ as the temperature is decreased. Hence, the detailed balance factor $\cosh ^{-2}\left(\epsilon / 2 k_{B} T\right) \leqslant 1$ only plays a minor role, and always suppresses the SR phenomenon (cf. Fig. 7). With $\exp \left(-\epsilon / k_{B} T\right) \ll 1$, i.e., $\bar{\Gamma} \simeq \Gamma_{+}$the power amplification $\widetilde{\eta}_{1}$ is exponentially reduced proportionally to $\left[\exp \left(-\epsilon / k_{B} T\right)\right]^{2}$; likewise the SNR is exponentially (but more weakly) reduced proportionally to $\exp \left(-\epsilon / k_{B} T\right)$. This finding is in accordance with prior studies of SR in nonequilibrium systems [31].

(v) On the other hand, in the deep quantum regime, the decay rates exhibit a smoother (non-Arrenhius) temperature dependence and remain finite even at zero temperature [17]. Further, within a two-state description of the incoherent tunneling dynamics, the energy splitting of the two discrete energy levels is of the order of the asymmetry energy $\epsilon$. Hence, the detailed balance factor represents the relative occupation of the energy levels and plays a crucial role. Whenever $\epsilon \ll k_{B} T$ the energy levels are almost equally occupied, so that the limit $\epsilon=0$ yields no SR phenomenon [22,23].

\section{QUANTUM ENHANCEMENT OF SR}

We now apply the linear-response results discussed in the preceding sections, valid for weak external signals, to the study of the semiclassical SR. Hence, the study of SR in the temperature regime where quantum corrections to the classical rate of activation are important reduces to the evaluation of the semiclassical escape rates $\Gamma_{+}$or $\Gamma_{-}$at thermal equilibrium. The thermal escape rate $\Gamma$ out of a single metastable state can be evaluated using the thermodynamic method (the quantum transition-state theory) first proposed by Langer [32], or by an equivalent periodic orbit approach [16]. Following Langer, above $T_{0}$ the escape rate is related to the 
imaginary part $\operatorname{Im} F$ of the free energy of the metastable system by the relation $\Gamma=-(2 / \hbar)\left(T_{0} / T\right) \operatorname{Im} F$, and below $T_{0}$ by $\Gamma=-(2 / \hbar) \operatorname{Im} F$. This method is, in fact, not restricted to the classical regime, because one can study as well the quantum partition function of the system. When the potential is not metastable but consists of two wells, as in Fig. 1, the Langer method will yield the backward and forward rates as long as there is no phase coherence between the "reactant" and "product" states. In particular, as shown in Ref. [30], the $\operatorname{Im} F$ method can be justified for dissipative quantum tunneling as long as only incoherent tunneling occurs. However, because Langer's method requires thermal equilibrium with the environment, it does not extend to the region of energydiffusion-limited classical escape, occurring for extremely underdamped systems $\hat{\gamma}\left(\omega_{b}\right) \leqslant \omega_{b} k_{B} T / V_{b}$ with $\hat{\gamma} \ll \omega_{b}$. Such an extension is possible by use of the quantum kinetic turnover approach put forward in [17]. In the following, however, we shall restrict the discussion to moderate-to-large friction such that the quantum transition-state theory always holds.

Starting from a path-integral formulation, the free energy can be evaluated semiclassically using a steepest-descent approximation whenever $V_{b} \gg \hbar \omega_{R}[13-15,30]$. The crossover temperature $T_{0}$ is then just the temperature below which the lowest energy fluctuation mode around the classical path $q(\tau)=q_{b}$ in the inverted metastable potential $-V(q)$ becomes unstable. This instability indicates the appearance of an additional solution that becomes the dominant (stable) one below $T_{0}$. Just below $T_{0}$, this new solution is a periodic (with period $\hbar \beta$ ) oscillation of small amplitude about the minimum of the inverted metastable potential, called "bounce." As the temperature is further lowered, the bounce solution evolves in a way that depends on the nonlinearity of the potential. For the double-well potential, the bounce solution evolves far below $T_{0}$ into trajectories called "instantons" where the particle starts from the bottom of one well, traverses the classically forbidden region between the two wells repeatedly, and finally returns to the starting point $[30,17]$. Hence, the appearance of the bounce solution is interpreted as the appearance of a new channel for barrier crossing (quantum tunneling) which dominates at very low temperatures. Above $T_{0}$, the presence of the additional quantum channel for barrier crossing at thermal equilibrium results in a quantum correction factor $f_{q}$ that merely multiplies the classical rate of activation [13-15,33],

$$
\Gamma=f_{q} \Gamma_{\mathrm{cl}},
$$

where

$$
f_{q}=\prod_{n=1}^{\infty} \frac{+\omega_{a}^{2}+n^{2} \nu^{2}+n \nu \hat{\gamma}(n \nu)}{-\omega_{b}^{2}+n^{2} \nu^{2}+n \nu \hat{\gamma}(n \nu)}
$$

and $\nu=2 \pi k_{B} T / \hbar$. The factor $f_{q}$ approaches unity for $T \gg T_{0}$ and diverges exactly at the crossover temperature $T_{0}$. This divergence can be regularized taking into account the deviation of the barrier top from the parabolic form $[14,17]$; however, the regularization is only necessary in a very small region in the vicinity of $T_{0}$ (crossover region in Fig. 2). Because, as we shall see, the maxima in the SNR and in $\eta_{1}$ appear well above $T_{0}$, for our purposes the regulariza- tion is not needed. At temperatures $T \gg \hbar \omega_{a} / k_{B}$, the leading quantum corrections are found to be given by the hightemperature approximation $[14]$

$$
f_{q}=\exp \left[\frac{\hbar^{2}}{24}\left(\omega_{a}^{2}+\omega_{b}^{2}\right) /\left(k_{B} T\right)^{2}\right],
$$

being independent of the dissipative mechanism. Thus, the overall effect of the quantum fluctuations is to facilitate the escape because they increase the average energy of the particle in the metastable wells [the $\omega_{a}^{2}$ contribution in (3.3)] and because, for a particle that is almost thermally excited up to the barrier top, they allow for tunneling through the remaining barrier region $\left[\omega_{b}^{2}\right.$ contribution in (3.3)]. As shown by Eq. (3.3), both effects result in an effective reduction of the barrier and, correspondingly, in an exponential enhancement of the relaxation rate.

So far we have considered arbitrary frequency-dependent damping. In the following we shall focus on the case of frequency-independent Ohmic damping where the product (3.2) can be evaluated exactly in terms of gamma functions $\Gamma[33]$ :

$$
f_{q}=\frac{\Gamma\left(1-\lambda_{0}^{+} / \nu\right) \Gamma\left(1-\lambda_{0}^{-} / \nu\right)}{\Gamma\left(1-\lambda_{a}^{+} / \nu\right) \Gamma\left(1-\lambda_{a}^{-} / \nu\right)},
$$

where, introducing the dimensionless coupling parameter $\alpha=\gamma / 2 \omega_{b}$, the frequencies $\lambda_{0}^{ \pm}, \lambda_{a}^{ \pm}$are

$$
\begin{gathered}
\lambda_{0}^{ \pm}=-\omega_{a}\left[\alpha \pm\left(\alpha^{2}+1\right)^{1 / 2}\right], \\
\lambda_{a}^{ \pm}=-\omega_{b}\left[\alpha^{2} \pm\left(\alpha^{2}-\left(\omega_{a} / \omega_{b}\right)^{2}\right)^{1 / 2}\right] .
\end{gathered}
$$

It is now interesting to observe that for strongly damped systems $\alpha \gg\left\{1, \omega_{a} / \omega_{b}\right\}$ and intermediate temperatures $T_{0} \ll T \ll 4 \alpha^{2} T_{0}$, the above Eq. (3.4) simplifies to [15]

$$
f_{q}=\left(4 \alpha^{2} T_{0} / T\right)^{\left(1+\omega_{a}^{2} / \omega_{b}^{2}\right) T / T_{0}},
$$

so that the rate can be enhanced substantially even well above the crossover temperature. For temperatures $T \gg 4 \alpha^{2} T_{0}$, the factor $f_{q}$ is again approximated by the hightemperature expression Eq. (3.3). A final remark concerns the conditions $V_{b} \gg \hbar \omega_{R}$ and $V_{b} \gg k_{B} T$ that ensure the validity of the semiclassical approximation and of separation of time scales, respectively. Because $\omega_{R} \approx \omega_{b}$ for weakly damped systems and $\omega_{R} \approx \omega_{b} / \alpha$ for strongly damped systems [cf. Eq. (1.4)], it is apparent that the potential barrier can be very small compared to the scale $\hbar \omega_{b}$ when the system is heavily damped. On the other hand, for strongly damped systems the condition of well separated time scales amounts to $V_{b} \gg\left(T / T_{0}\right)\left(\hbar \omega_{b} / 4 \pi \alpha\right)$. The fulfillment of these conditions has been checked self-consistently in our numerical results. Further, because, as shown by Eq. (3.3), the leading quantum corrections at high temperatures are of order $(\hbar \beta)^{2}\left(\omega_{a}^{2}+\omega_{b}^{2}\right)$, to be consistent with the semiclassical approximation to the linear susceptibility of Eq. (2.19) we have to require that $2 \Omega^{2} \ll \omega_{a}^{2}+\omega_{b}^{2}$.

In Figs. 3-6 we discuss our results for a symmetric double-well potential $(\epsilon=0)$, where the SR phenomenon for 
the semiclassical and classical $\widetilde{\eta}_{1}$ (but also for $\widetilde{\mathcal{R}}$ ) is maximal (cf. Fig. 7). The semiclassical scaled signal-to-noise ratio $\widetilde{\mathcal{R}^{s c}}$ and scaled power amplitude $\widetilde{\eta}_{1}^{s c}$ are investigated in Figs. 3 and 4, respectively, for strong Ohmic coupling $\alpha=50$. We assume $\omega_{b}=\omega_{a}$ and barrier height $V_{b}=0.2 \hbar \omega_{b}$. For comparison also the classical (i.e. $\hbar=0$ ) quantities $\widetilde{\mathcal{R}}^{c l}$ and $\widetilde{\eta}_{1}^{c l}$ are plotted (dashed line). It is now apparent that for strongly damped systems and not too high barriers, quantum fluctuations strongly influence the resulting output signal, as compared with the results of a classical analysis. In particular, the amplitude of the maximum is enhanced by quantum corrections and the position of the maximum itself is shifted towards lower temperatures. As shown in the insets of Figs. 3 and 4, in the proximity of the crossover temperature, the enhancement can even exceed two orders of magnitude. As the coupling constant $\alpha$ is increased, for fixed barrier height the position of the maximum of $\widetilde{\eta}_{1}$ $(\widetilde{\mathcal{R}})$ is shifted toward higher temperatures. On the other hand, because for strongly damped systems quantum effects persist well above $T_{0}$ [cf. Eq. (3.6)], the semiclassical SR can still differ appreciably from the classical SR. This is shown in Fig. 5, where the power amplitude $\widetilde{\eta}_{1}$ is depicted for different values of the Ohmic coupling constant $\alpha$. We choose $\alpha=50,75,100$ (left to right). The solid lines correspond to the semiclassical power amplitude $\widetilde{\eta}_{1}^{s c}$ and the dashed lines to the classical approximation $\widetilde{\eta}_{1}^{c l}$. For higher potential barriers and fixed damping, the position of the temperature maximum in $\widetilde{\eta}_{1}^{s c}$ or $\widetilde{\mathcal{R}}^{s c}$ moves toward higher temperatures and the system behaves classically. In other words, the quantum-corrected lines merge into the classical approximation. As previously mentioned, the position of the temperature maximum $T_{\eta_{1}}^{*}$ of $\widetilde{\eta}_{1}^{s c}$ depends also on the applied external frequency. This result is shown in Fig. 6, where $T_{\eta_{1}}^{*}$ becomes shifted toward lower temperatures as the driving frequency $\Omega$ is decreased (the curves are for $\Omega / \omega_{b}=10^{-6}$, $\left.10^{-5}, 10^{-4}\right)$. In the inset, the ratio $\bar{\Gamma}\left[T_{\eta_{1}}^{*}(\Omega)\right] / \Omega$ is depicted for five chosen frequency values. It is remarkable that within four orders of magnitude for $\Omega$ the ratio is approximately constant and of order unity. Hence, the semiclassical SR maximum appears roughly at the temperature $T_{\eta_{1}}^{*}$ at which the semiclassical rate $\bar{\Gamma}\left(T_{\eta_{1}}^{*}\right)$ is at resonance with the driving frequency $\Omega$ of the coherent external force. Finally, as discussed above, $\widetilde{\eta}_{1}$ is plotted in Fig. 7 versus the asymmetry $\epsilon$ of the potential shown in Fig. 1, where we use $V^{\prime \prime}\left(q_{a}\right)=V^{\prime \prime}\left(-q_{a}\right)=M \omega_{a}^{2}$. As in the classical case (dashed line) [31], the semiclassical power amplitude (solid line) is maximal for symmetric systems.

\section{CONCLUSIONS}

In conclusion, we investigated the phenomenon of SR in relation to the complicated interplay between quantum and thermal fluctuations. Because the thermal escape rate can be strongly enhanced in the presence of the additional quantum tunneling channel, we showed that both the signal-to-noise ratio $\mathcal{R}$ and the power amplitude $\eta_{1}$ can be strongly amplified by quantum fluctuations (e.g., up to 300 times; cf. the insets of Figs. 3 and 4). As a difference compared to the classical case, in a semiclassical analysis the effects of temperature and dissipation on the escape rate have to be considered separately. Hence, our analysis represents the quantum generalization of SR for the Kramers equation [34], thereby covering moderate-to-large friction on a unified basis.

The value itself of the crossover temperature $T_{0}$, where quantum transitions dominate over tunneling events, strongly depends on the friction coefficient, especially for heavily damped systems. In particular, for strongly damped systems, we showed that the effects of quantum fluctuations on SR can extend well above $T_{0}$. At fixed friction, quantum effects become successively washed out for systems with increasing barrier heights.

Possibilities for observing the predicted tunneling corrections to classical SR are abundant. These concern all those systems where quantum corrections to the thermal escape rate could be established (see Sec. XI of Ref. [17]). Application of a small periodic ac perturbation then allows one to study these SR quantum corrections. A particularly suitable experimental system is a SQUID, where quantum corrections to the escape rate [20], and very recently the phenomenon of classical SR [19] itself, have been observed.

\section{ACKNOWLEDGMENTS}

M.G. and P.H. gratefully acknowledge the support of this work by the Deutsche Forschungsgemeinschaft (HA1517/14$1)$.
[1] R. Benzi, A. Sutera, and A. Vulpiani, J. Phys. A 14, L453 (1981); C. Nicolis, Sol. Phys. 74, 473 (1981).

[2] See, for example, Proceedings of the Nato Workshop on SR in Physics and Biology, St. Diego, 1992 [J. Stat. Phys. 70, 1 (1993)].

[3] For reviews, see, F. Moss, D. Pierson, and D. O'Gorman, Int. J. Bifurc. Chaos 4, 1383 (1994); P. Jung, Phys. Rep. 234, 175 (1993).

[4] J. Maddox, Nature 369, 271 (1994); J. K. Douglass, L. Wilkens, E. Pantazelou, and F. Moss, Nature 365, 337 (1993); K. Wiesenfeld and F. Moss, Nature 373, 33 (1995).
[5] B. McNamara, K. Wiesenfeld, and R. Roy, Phys. Rev. Lett. 60, 2626 (1988).

[6] P. Jung and P. Hänggi, Europhys. Lett. 8, 505 (1989); Phys. Rev. A 44, 8032 (1991).

[7] L. Gammaitoni, F. Marchesoni, E. Menichelli-Saetta, and S. Santucci, Phys. Rev. Lett. (1989); L. Gammaitoni, E. Menichella-Saetta, S. Santucci, F. Marchesoni, and C. Presilla, Phys. Rev. A 40, 2114 (1989).

[8] B. McNamara and K. Wiesenfeld, Phys. Rev. A 39, 4854 (1989).

[9] R. Fox and Y. Lu, Phys. Rev. E 48, 3390 (1993). 
[10] R. F. Grote and J. T. Hynes, J. Chem. Phys. 73, 2715 (1980); P. Hänggi and F. Mojtabai, Phys. Rev. A 26, 1168 (1982); J. Stat. Phys. 30, 401 (1983).

[11] H.A. Kramers, Physica (Utrecht) 7, 284 (1940).

[12] V. I. Gol'danskii, Dok. Akad. Nauk SSSR 124, 1261 (1959) [Sov. Phys. Dokl. 4, 1172 (1959)]; 127, 1037 (1959).

[13] H. Grabert and U. Weiss, Phys. Rev. Lett. 53, 1787 (1984); H. Grabert, in SQUID '85, edited by H.D. Hahlbohm and H.L. Lübbig (de Gruyter, Berlin, 1985).

[14] P. Hänggi, H. Grabert, G.L. Ingold, and U. Weiss, Phys. Rev. Lett. 55, 761 (1985).

[15] H. Grabert, P. Olschowski, and U. Weiss, Phys. Rev. B 36, 1931 (1987).

[16] P. Hänggi and W. Hontscha, Ber. Bunsenges. Phys. Chem. 95, 379 (1991).

[17] (a) P. Hänggi, P. Talkner, and M. Borkovec, Rev. Mod. Phys. 62, 251 (1990); (b) P. Hänggi, in Activated Barrier Crossing, edited by G. R. Fleming and P. Hänggi (World Scientific, London, 1993).

[18] J. P. Bouchland, E. Cohen de Lara, and R. Kahn, Europhys. Lett. 17, 583 (1992).

[19] R. Rouse, S. Han, and J.E. Lukens, Appl. Phys. Lett. 66, 108 (1995); A. D. Hibbs, A. L. Singaas, E. W. Jacobs, A. R. Bulsara, J. J. Bekkedahl, and F. Moss, J. Appl. Phys. 77, 2582 (1995).

[20] D. B. Schwartz, B. Sen, C. N. Archie, and J. E. Lukens, Phys. Rev. Lett. 55, 1547 (1985); ibid. 57, 266 (1986); H. Grabert, P. Olschowski, and U. Weiss, Phys. Rev. Lett. 57, 265 (1986).

[21] J. Clarke, A. N. Cleland, M. H. Devoret, D. Esteve, and J. M. Martinis, Science 239, 992 (1988).

[22] R. Löfstedt and S. N. Coppersmith, Phys. Rev. Lett. 72, 1947 (1994); Phys. Rev. E 49, 4821 (1994).

[23] M. Grifoni and P. Hänggi, Phys. Rev. Lett. 76, 1611 (1996);
Phys. Rev. E (to be published).

[24] A. J. Leggett, S. Chakravarty, A. T. Dorsey, M. P. A. Fisher, A. Garg, and W. Zwerger, Rev. Mod. Phys. 59, 1 (1987).

[25] U. Weiss, Quantum Dissipative Systems, Series in Modern Condensed Matter Physics, Vol. 2 (World Scientific, Singapore, 1993).

[26] In Eq. (2.4) we dropped a term that depends on the initial condition for the particle and decays on a time scale given by the memory time of the reservoir.

[27] It should be noted that, due to the explicit time dependence of the perturbation, the correlation function $\langle q(t+\tau) q(t)$ $+q(t) q(t+\tau)\rangle / 2$ depends separately on the time arguments $t$ and $\tau$. The time-averaged function $\bar{C}(\tau)$ is again only a function of the time difference $\tau$ [6].

[28] The quantum FDT relates the power spectrum in thermal equilibrium $S_{N}^{(0)}=S_{N}(A=0)$ to the linear susceptibility via the relation $S_{N}^{(0)}(\Omega)=\hbar \operatorname{coth}(\beta \hbar \Omega / 2) \operatorname{Im} \widetilde{\chi}(\Omega)$. In the limit $\hbar \beta \Omega / 2 \ll 1$ the FDT becomes $S_{N}^{(0)}(\Omega)=2 \operatorname{Im} \widetilde{\chi}(\Omega) / \beta \Omega$ and equals the classical FDT.

[29] U. Weiss and H. Grabert, Phys. Lett. 108A, 63 (1985).

[30] U. Weiss, H. Grabert, P. Hänggi, and P. Riseborough, Phys. Rev. B 35, 8535 (1987).

[31] R. Bartussek, P. Hänggi, and P. Jung, Phys. Rev. E 49, 3930 (1994); R. Bartussek, P. Jung, and P. Hänggi, in Noise and Physical Systems and 1/f Fluctuations, edited by P. H. Handel, AIP Conf. Proc. No. 285 (AIP, New York, 1993), p. 661.

[32] J. S. Langer, Ann. Phys. (NY) 41, 108 (1967).

[33] P. G. Wolynes, Phys. Rev. Lett. 47, 968 (1981); V. I. Mel'nikov and S. V. Meshkov, Pis'ma Zh. Éksp. Teor. Fiz. 38, 111 (1983) [JETP Lett. 38, 130 (1983)]; E. Pollak, Chem. Phys. Lett. 127, 178 (1986).

[34] P. Hänggi, P. Jung, C. Zerbe, and F. Moss, J. Stat. Phys. 70, 25 (1993). 